\title{
Two-Photon Quantum Dot Excitation during Optical Trapping
}

\author{
Liselotte Jauffred, and Lene B. Oddershede* \\ The Niels Bohr Institute, University of Copenhagen, Denmark
}

\begin{abstract}
A single CW infrared laser beam can simultaneously trap and excite an individual colloidal quantum dot. Though the laser light is relatively weak, the excitation occurs through two-photon absorption. This finding eliminates the demand for an excitation light source in addition to a trapping laser in nanoscale experiments with simultaneous force-manipulation and quantum dot visualization. Also, we demonstrate that optical trapping efficiencies of individual quantum dots do not correlate with their emission wavelength or physical size.
\end{abstract}

KEYWORDS Quantum dots, optical trapping, two-photon-absorption, fluorescence microscopy, nanoparticles

W ith their extreme luminescence and bleaching properties, quantum dots (QDs) are desirable to use as fluorescent markers on biological specimen or nanoscale materials. ${ }^{1-4}$ Optical tweezers can trap and manipulate particles and there are continuous efforts to push the lower size limit on the manipulated particles. Metallic nanoparticles with dimensions down to $10 \mathrm{~nm}$ have been proven individually manipulated. ${ }^{5-9}$ Aggregates of QDs have been trapped with pulsed high power lasers ${ }^{10}$ and even individual QDs were trapped with CW infrared optical tweezers. ${ }^{11}$ This is very useful, as a single QD can serve both for visualization and as a handle for controlled force transduction. Quantum dots can be delivered into living cells ${ }^{12}$ and attached to individual proteins, ${ }^{13}$ and hence, a combined visualization and force manipulation of quantum dots hold promise of quantifying intracellular nanomechanics. The QD can become excited either by absorbing one photon or by absorbing two photons simultaneously, two-photon absorption being significantly less likely to occur. Observations of two-photon absorption by colloidal QDs are relatively sparse but have been reported to occur using high-energy pulsed lasers with a peak power of $3 \times 10^{10} \mathrm{~W} / \mathrm{cm}^{2} .{ }^{14,15}$ However, high-energy pulsed lasers are potentially harmful to biological assays. Here, we report that QDs can even become excited by two-photon absorption of relatively weak CW infrared laser light $\left(100 \mathrm{~mW} / \mu \mathrm{m}^{2} \sim 10^{7} \mathrm{~W} / \mathrm{cm}^{2}\right)$ and that the laser light simultaneously is able to trap the QDs. Also, we demonstrate how the physical constants characterizing the strength of the optical trap correlate with the physical size and emission color of individual QDs and how the twophoton absorption affects the overall polarizability of the QD.

A variety of water-soluble streptavidin-coated QDs (Invitrogen) with emission wavelengths $(\lambda) 525,585,605,655$, 705 , and $800 \mathrm{~nm}$ were used for the experiments. The QDs

* To whom correspondence should be addressed. E-mail: oddershede@nbi.dk. Received for review: 03/16/2010

Published on Web: 04/19/2010 had CdSe or CdSeTe cores and thin ZnS shells and were stabilized with an outer layer of polymers and PEG. ${ }^{17}$ There is no detectable difference in optical trapping properties of individual QDs uncoated or coated with streptavidin. ${ }^{11}$ As most QDs in nanobioassays are coated to make a specific attachment possible, we carried through the present investigation using streptavidin coated QDs. We used a $50 \mathrm{mM}$ sodium borate ( $\mathrm{pH}$ 8.2) solution with $1 \%$ BSA to maximize stabilization and minimize aggregation. ${ }^{11}$ Two types of experiments were carried out involving optical trapping and investigations of (1) aggregates of QDs or (2) individual QDs. For type (1) experiments, the QDs were diluted $1 / 10^{2}-1 /$ $10^{3}$ into the solution. For the type (2) experiments, the QDs were diluted $1 / 10^{5}$ into the solution and the solution was pressed through a filter to remove aggregates. Presence of an individual QD in the trap could be monitored through time series analysis, ${ }^{11}$ and the concentration was adjusted such that there was a typical waiting time of several minutes before an additional QD would enter the trap.

The trapping laser was a $\mathrm{Nd}: \mathrm{YVO}_{4}$ laser $(5 \mathrm{~W}$ Spectra Physics Millennia, $1064 \mathrm{~nm}, \mathrm{TEM}_{00}$ ) implemented in an inverted Leica microscope. A quadrant photodiode (S5981, Hamamatsu), allowed for precise position detection. A Hg lamp was used for linear excitation of the QDs. The laser was focused to a diffraction limited spot by an oil immersion objective (Leica HCX PL Apo, 63×, NA $=1.32, \infty, 0.17$ ).

To maximize the strength of the trap in the axial direction we used an immersion oil with a refractive index of $n=1.54$ (Cargille), which efficiently canceled spherical aberrations at a depth of $5 \mu \mathrm{m} .{ }^{16}$ However, this immersion oil turned out to be fluorescent. Hence, for type (1) experiments we used an oil objective (Leica HCX PL APO 100× NA $=1.4$ oil CS) and a nonfluorescent immersion oil (Leica with $n=1.518$ ). To trap and simultaneously visualize the $605 \mathrm{~nm}$ QD, we used a custom-made filter cube (Leica HQ420/40x + 1064, z440/1065rpc, HQ605/40 m-2p), which allowed the $420 \mathrm{~nm}$ light from the Hg lamp as well as the $1064 \mathrm{~nm}$ laser light to 


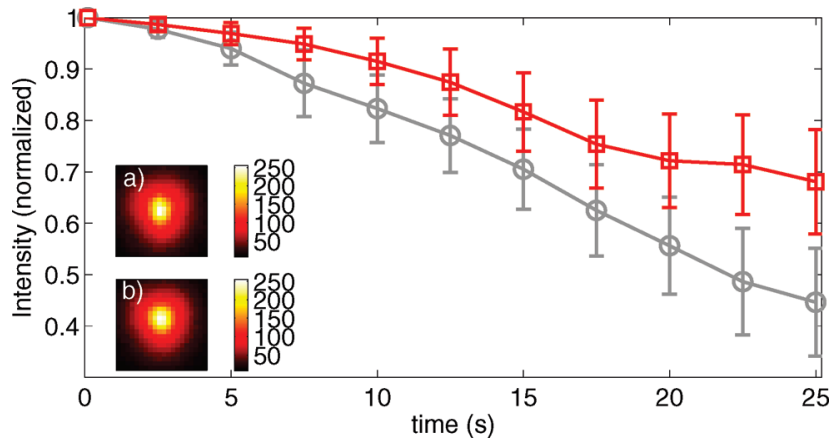

FIGURE 1. Average value of normalized emission from trapped QD aggregates $(\lambda=605 \mathrm{~nm})$ as a function of time. Upper curve (red squares) shows emission with laser on (Hg lamp off). Lower curve (gray circles) shows emission with both laser and Hg lamp on. Error bars denote SEM. Picture (a) shows emission from a trapped aggregate (no Hg lamp). Picture (b) shows emission from a trapped aggregate with the Hg lamp on.

be reflected up to the sample and only allowed the emitted $605 \mathrm{~nm}$ light to be transmitted to the EMCCD (Ixon, Andor).

With the filter cube it was possible to simultaneously trap and visualize $605 \mathrm{~nm}$ QDs, regardless of whether the excitation source was the Hg lamp or the trapping laser alone. The picture shown in Figure 1a is the emission from an aggregate of 605 QDs trapped by the $1064 \mathrm{~nm}$ laser. The Hg lamp is turned off. As there are no photons available with wavelengths below $605 \mathrm{~nm}$, this picture is the first proof of $\mathrm{QD}$ excitation mediated by two-photon absorption of the trapping laser light. Figure $1 \mathrm{~b}$ is another optically trapped $\mathrm{QD}$ aggregate, but in this picture the Hg lamp is on. Hence, the emitted signal is a result of both one-photon and two-photon excitation.

Traces showing the emission signal/noise $(S / N)$ as a function of time for individual aggregates are shown in Supporting Information Figure 1. The exact number of QDs in one aggregate was not known, but the total intensity at the beginning of the trace probably reflects this number. Individual curves show a stepwise behavior, each step possibly signifying the bleaching of an individual QD in the aggregate. The pictures were taken at a sampling rate of $10 /$ $\mathrm{s}$, thus integrating over several on states of the QDs in each frame. The curves in Figure 1 shows the average values of the emitted intensity as a function of time. As the aggregates consisted of a varying number of QDs, the intensity is normalized by the first value. The upper curve (red squares) is the result of two-photon excitation alone (no Hg lamp), the lower curve (gray circles) is the result of both two-photon and one-photon excitation (Hg lamp on). It is clear that the aggregates undergo bleaching over a time scale of $25 \mathrm{~s}$ and that bleaching is faster when both two-photon and onephoton excitation takes place.

Interestingly, we did not detect emission from the 525 $\mathrm{nm}$ QD while trapped by the $1064 \mathrm{~nm}$ laser light (no Hg lamp). This is reasonable, because two-photon absorption requires the total energy of the photons to be at least equal to the energy, $E$, corresponding to the emission wavelength,

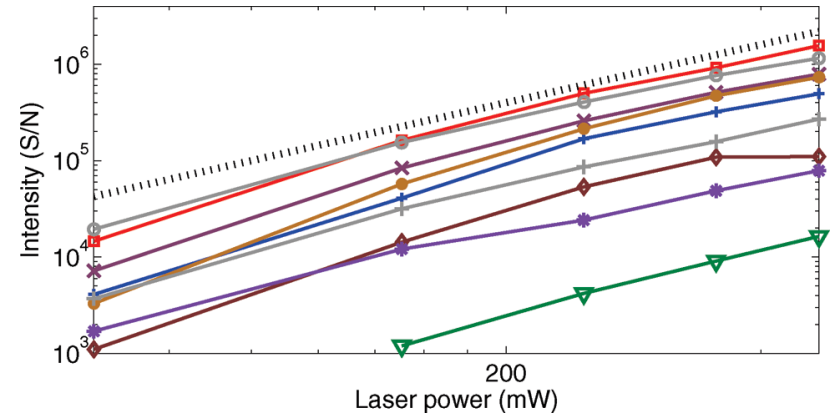

FIGURE 2. Emitted intensity (signal/noise) versus laser power for individual $\mathrm{QD}$ aggregates of varying initial size. The dotted line has a slope of 2 .

$\lambda$, of the $\mathrm{QD}, E=(c h) /(\lambda)$, where $c$ is the speed of light and $h$ is Planck's constant. However, we did not observe $1064 \mathrm{~nm}$ induced emission from a $585 \mathrm{~nm} \mathrm{QD}$. This might be because the absorption of this QD is relatively low at $532 \mathrm{~nm}$ (as supported by the absorption spectrum of $585 \mathrm{~nm}$ QDs available at Invitrogen's Web site). The $605 \mathrm{~nm}$ QD and all QDs with $\lambda$ above have a relatively high absorption of 532 $\mathrm{nm}$ light and all exhibit a clear and visible $1064 \mathrm{~nm}$ induced two-photon excitation.

To further prove the existence of two-photon-absorption of a QD that is trapped by the exciting laser beam we monitored the emitted intensity as a function of laser power, $P$. For a two-photon absorption, the emitted intensity, $I$, should scale as $I \propto P^{2}$ (dotted line in Figure 2). Figure 2 shows the intensity of the emitted light from various trapped $Q D$ aggregates as a function of $P$. Regardless of initial aggregate size, all traces exhibit a scaling behavior characteristic of two-photon absorption.

It is important to correctly choose the QD such that it matches the given experimental goals and conditions, for example, excitation lasers and filter cubes. The emitted wavelength is dependent on the physical size of the QD. Therefore, we set out to investigate whether the trapping properties of individual QDs would vary as a function of physical size. All QDs in this present study were bought from Invitrogen who also provided the following information: ${ }^{17}$ the emission wavelength, which is intimately related to the size and shape of the core region, the composition, and the total diameter $(d)$ including the thin shell and the polymer coat. All these values are given in Table 1. As a check of the external dimensions and the quality of the stock we also made TEM pictures of the QDs, see examples in Figure 3a,b. We found the size distributions to be reasonably uniform and in agreement with the values from Invitrogen; our values are given in Table 1 also. However, as shown in Figure 3b, we found the 655 QD to have an overall elongated shape, which was not reported by Invitrogen, and the $585 \mathrm{~nm}$ QD was larger than expected.

From knowledge of the size of an individual QD it is possible to perform a quantitative analysis of its interaction with the electromagnetic field created by the optical trap. 
TABLE 1. Physical Characteristics of Investigated QDs ${ }^{a}$

\begin{tabular}{cllcl}
\hline $\begin{array}{c}\text { wavelength } \\
{[\mathrm{nm}]}\end{array}$ & $\begin{array}{c}\text { core } \\
d[\mathrm{~nm}]\end{array}$ & composition & $\begin{array}{c}\text { total } \\
\text { [nm] }\end{array}$ & $\begin{array}{c}\text { total } d \\
(\mathrm{TEM})[\mathrm{nm}]\end{array}$ \\
\hline 525 & $3-4$ & CdSe/ZnS & 13 & $10 \pm 2$ \\
585 & 5.3 & CdSe/ZnS & 15 & $26 \pm 12$ \\
605 & $(4 \times 9.4)$ & CdSe/ZnS & 16 & $13 \pm 1$ \\
655 & $(6 \times 12)$ & CdSe/ZnS & 20 & $(40 \pm 5) \times(24 \pm 3)$ \\
705 & ND & CdSeTe/ZnS & 20.5 & $16 \pm 3$ \\
800 & ND & CdSeTe/ZnS & 21 & $21 \pm 3$
\end{tabular}

${ }^{a}$ First column gives emission wavelength, second column shows the size of the core region, which is given by the diameter if the QDs are spherical or by the semimajor and semiminor axes where the QDs are more ellipsoidal, third column states the material composition, and the forth column gives $d$, core plus shell plus polymer coat (all numbers from Invitrogen). The last column gives the outer $d$ as measured by TEM.
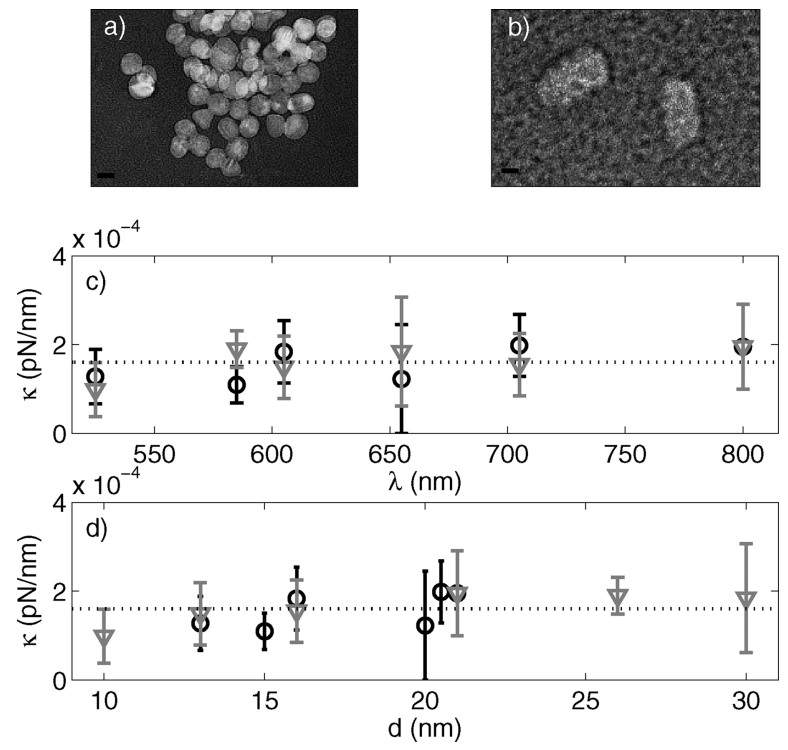

FIGURE 3. TEM pictures of (a) $605 \mathrm{~nm}$ QDs, (b) $655 \mathrm{~nm}$ QDs, the scale bars are $10 \mathrm{~nm}$. (c) Trapping spring constant of an individual QD as a function of (c) emission wavelength (d) total diameter. Black circles denote $\kappa$ based on $d$ given by Invitrogen, gray triangles are based on TEM measurements of $d$. Error bars denote one SD. The dotted line is the average value $\kappa$.

Optical tweezers exert a harmonic force on a trapped particle: $\mathbf{F}=-\kappa x$, where $\kappa$ denotes the trap stiffness and $\boldsymbol{x}$ is the position of the particle with respect to the center of the trap. The equation of motion of a particle performing Brownian fluctuations inside an optical trap is given by the Langevin equation. Fourier transformation of the Langevin equation gives a positional power spectrum which follows a Lorentzian function. As shown in ref 11, the power spectrum of an individual QD follows a Lorentzian function as expected. The frequency in the power spectrum that distinguishes the plateau region of slow fluctuations from the region with a slope of -2 (signifying Brownian motion for rapid fluctuations) is denoted the corner frequency, $f_{\mathrm{c}} . f_{\mathrm{c}}$ is related to $\kappa$ and to the drag coefficient, $\gamma$, of the $\mathrm{QD}, f_{\mathrm{c}}=$ $\kappa /(2 \pi \gamma)$. We determined $\gamma$ by Stokes law, $\gamma=3 \pi \eta d$, where $\eta$ denotes the viscosity of water. Hence, by fitting a Lorent-

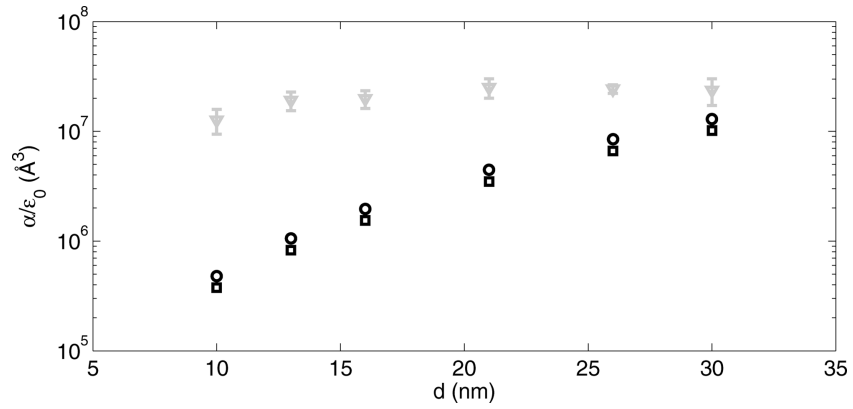

FIGURE 4. Polarizability versus QD diameter. Gray triangles denote experimentally determined values, error bars are SEM. The theoretical estimates are plotted using black squares (based on a linear refractive index) or black circles (based on a nonlinearly corrected refractive index).

zian function to the power spectrum, $f_{\mathrm{c}}$ can be found and through knowledge of $d, \kappa$ can be obtained.

For each type of $\mathrm{QD}, f_{\mathrm{c}}$ was found for at least 20 individual QDs and the laser power was kept at $100 \mathrm{~mW}$ at the sample. Figure 3c shows the trap stiffness, $\kappa$, as a function of $\mathrm{QD}$ emission wavelength, $\lambda$. Figure $3 d$ shows $\kappa$ as a function of $d$. As the values of $d$ from Invitrogen deviate somewhat from the TEM determined values, we depicted $\kappa$ using both values of $d$ (with black circles and gray triangles, respectively). The trap stiffness appears constant, independent of $\lambda$ or $d$, with an average value of $(1.6 \pm 0.4) \times 10^{-4} \mathrm{pN} / \mathrm{nm}$ (mean $\pm \mathrm{SD}$ ). This number is the same regardless of whether the Invitrogen or TEM determined values of $d$ are used, and within the error bars it coincides with the value previously reported for an individual $655 \mathrm{~nm}$ QD. ${ }^{11}$ The two-photon absorption cross section of CdSe QDs has been shown to correlated with the physical size of the QD. ${ }^{18}$ However, this effect is not large enough that we, within the uncertainty of the experiment, can detect a size dependence of the trapping strength.

In ref 11 , the polarizability of an individual QD was inferred from the measurement of $\kappa$ (denoting optical trapping strength in a direction perpendicular to the propagating laser light). The relation between the polarizability $\alpha$ and the laser power at the sample, $P$, was found to be

$$
\frac{\alpha}{\varepsilon_{0}}=\frac{2 \pi c \kappa \varepsilon}{P \varepsilon_{0}} \sigma^{4}
$$

where $\varepsilon$ is the electric permittivity of water, $\varepsilon_{0}=8.854 \times$ $10^{-12} \mathrm{C}^{2} /(\mathrm{Jm}), \sigma$ is the standard deviation of the Gaussian intensity distribution $(\sim 250 \mathrm{~nm}),{ }^{19}$ and $P=0.1 \mathrm{~W}$. Figure 4 (gray triangles) shows the experimentally determined values of $\alpha / \varepsilon_{0}$ for different sizes of QDs (estimated by TEM). The difference between these values of the polarization and the values reported in ref 11 stem from the different estimates of $d$.

The polarizability of a dielectric sphere can be theoretically estimated by the Claussius-Mossotti relation 


$$
\frac{\alpha}{\varepsilon_{0}}=3 V \frac{\epsilon_{\mathrm{qd}}-\epsilon}{\epsilon_{\mathrm{qd}}+2 \epsilon}
$$

where $\epsilon=\varepsilon / \varepsilon_{0}$ is the square of the refractive index $n$. For CdSe, $\epsilon_{\mathrm{qd}}=6.55^{20}$ and $\epsilon=1.74^{21}$ for water at $1064 \mathrm{~nm} . V$ is the volume of the $\mathrm{QD}$, which is found through knowledge of $d$ and by assuming a spherical shape. The fact that QDs absorb the trapping laser light hints that two-photon absorption should be taken into account when estimating the polarizability of individual QDs. Absorption leads to excitation of electrons within the QD, and the electrons in the conduction band are nearly free to respond to an applied electric field. ${ }^{22}$ Hence, the third-order nonlinear optical properties of CdSe and CdSe/ZnS core-shell QDs in solution give rise to nonlinear corrections of the index of refraction. ${ }^{23}$ The corrected index of refraction, $n_{c}$, can be written

$$
n_{\mathrm{c}}=n+\gamma I
$$

where $n$ is the linear refractive index and $\gamma$ is denoted the nonlinear refractive index. For CdSe illuminated with a wavelength of $1.06 \mu \mathrm{m}, \gamma$ is found experimentally to be $-1.47 \times 10^{-30} \mathrm{~W} \mu \mathrm{m}^{-2} \cdot{ }^{20}$ The theoretical estimates for polarizability are depicted in Figure 4, the black squares are based on simply using $n$ as the refractive index in eq 2 , and the black circles are based on using $n_{\mathrm{c}}$, which includes the nonlinear correction. As apparent from Figure 4, the absorption process that gives rise to the nonlinear contribution to the refractive index has only a minor effect on the total polarizability, $\alpha / \varepsilon_{0}$, found from eq 2 . One of the hallmarks of optical trapping of dielectric objects is a linear relationship between the spring constant and the trapping laser power. As shown in Supporting Information Figure 2, this is also true for optical trapping of two-photon excited QDs thus supporting that this nonlinear phenomenon is only a weak perturbation not altering trapping properties considerably.

We have shown that individual colloidal quantum dots can readily be excited by two-photon absorption of a relatively weak CW infrared laser that simultaneous traps the quantum dot. For an individual quantum dot, we investigated its interaction with the electromagnetic field. The trapping spring constant, $\kappa$, was independent of emission wavelength or physical size of the quantum dot. The physical size of each color of quantum dot was determined by TEM, and in two out of six colors the size or shape were significantly different than anticipated. Two-photon absorption gave rise only to a small correction of the total polarizability of an individual quantum dot. These results provide a basis for experimental design of future investigations using quantum dots and imply that only one CW laser is needed for experiments where quantum dots are to be used for nanoscale visualization, manipulation, or force transducers.

Acknowledgment. We thank technical assistant I. Jensen for producing the TEM images as well as C. B. Lagerholm, E. A. Christensen, and A. C. Richardson for fruitful discussions.

Supporting Information Available. Figures showing (1) intensity $(S / N)$ of individual aggregates, trapped by optical tweezers, over time, and (2) trapping strength versus laser power for quantum dots in the optical trap. This material is available free of charge via the Internet at http://pubs.acs.org.

\section{REFERENCES AND NOTES}

(1) Chan, W. C. W.; Nie, S. M. Science 1998, 281, 2016-2018.

(2) Michalet, X.; Pinaud, F.; Lacoste, T. D.; Dahan, M.; Bruchez, M. P.; Alivisatos, A. P.; Weiss, S. Single Mol. 2001, 2, 261-276.

(3) Michalet, X.; Pinaud, F.; Bentolila, L.; Tsay, J.; Doose, S.; Li, J.; Sundaresan, G.; Wu, A.; Gambhir, S.; Weiss, S. Science 2005, 307, 538-544.

(4) Larson, D.; Zipfel, W.; Williams, R.; Clark, S.; Bruchez, M.; Wise, F.; Webb, W. Science 2003, 300, 1434-1436

(5) Svoboda, K.; Block, S. M. Ann. Rev. 1994, 23.

(6) Bosanac, L.; Aabo, T.; Bendix, P. M.; Oddershede, L. B. Nano Lett. 2008, 8, 1486-1491.

(7) Hansen, P. M.; Bhatia, V. K.; Harrit, N.; Oddershede, L. Nano Lett. 2005, 5, 1937-42.

(8) Selhuber-Unkel, C.; Schubert, I. Z. O.; Sönnichsen, C.; Oddershede, L. B. Nano Lett. 2008, 8, 2998-3003.

(9) Hajizadeh, F.; Reihani, S. N. S. Opt. Express 2010, 18, 551-559

(10) Pan, L.; Ishikawa, A.; Tamai, N. Phys. Rev. B 2007, 75, 161305.

(11) Jauffred, L.; Richardson, A. C.; Oddershede, L. B. Nano Lett. 2008, 8, 3376-3380

(12) Yum, K.; Na, S.; Xiang, Y.; Wang, N.; Yu, M. Nano Lett. 2009, 9, 2193-2198.

(13) Ebenstein, Y.; Gassman, N.; Kim, S.; Antelman, J.; Kim, Y.; Ho, S.; Samuel, R.; Michalet, X.; Weiss, S. Nano Lett. 2009, 9, $1598-$ 1603 .

(14) Blanton, S.; Dehestani, A.; Lin, P.; Guyot-Sionnest, P. Chem. Phys. Lett. 1994, 229, 317-322

(15) Schmidt, M.; Blanton, S.; Hines, M.; Guyot-Sionnest, P. Phys. Rev. B 1996, 19, 12629.

(16) Reihani, S. N. S.; Oddershede, L. B. Opt. Lett. 2007, 32, $1998-$ 2000.

(17) Kraus, M. Personal communication about quantum dot sizes, Technical Report, Invitrogen, 2008.

(18) Pu, S.-C.; Yang, M.-J.; Hsu, C.-C.; Lai, C.-W.; Hsieh, C.-C.; Lin, S. H.; Cheng, Y.-M.; Chou, P.-T. Small 2006, 2, 308-1313.

(19) Svoboda, K.; Block, S. M. Opt. Lett. 1994, 19, 930-932.

(20) Sheik-Bahae, M.; Hutchings, D.; Hagan, D.; Stryland, E. V. IEEEJ. Quantum Electron. 1991, 27, 1296-1309.

(21) Segelstein, D. J. The complex refractive index of water. Master's Thesis, University of Missouri-Kansas City, Kansas City, MO, 1981.

(22) Boyd, R. Nonlinear Optics; Academic Press: New York, 2003.

(23) Gerdova, I.; Haché, A. Opt. Commun. 2005, 246, 205-212. 